\title{
Pharmacokinetic and metabolomic analyses of Mangiferin calcium salt in rat models of type 2 diabetes and non-alcoholic fatty liver disease
}

\author{
He Lin ${ }^{1 *}$, Houlei Teng ${ }^{2}$, Wei Wu², Yong Li ${ }^{1}$, Guangfu Lv' ${ }^{1}$ Xiaowei Huang ${ }^{1}$, Wenhao Yan ${ }^{1}$ and Zhe Lin ${ }^{1 *}$
}

\begin{abstract}
Background: Non-alcoholic fatty liver is one of the most common comorbidities of diabetes. It can cause disturbance of glucose and lipid metabolism in the body, gradually develop into liver fibrosis, and even cause liver cirrhosis. Mangiferin has a variety of pharmacological activities, especially for the improvement of glycolipid metabolism and liver injury. However, its poor oral absorption and low bioavailability limit its further clinical development and application. The modification of mangiferin derivatives is the current research hotspot to solve this problem.
\end{abstract}

Methods: The plasma pharmacokinetic of mangiferin calcium salt (MCS) and mangiferin were monitored by HPLC. The urine metabolomics of MCS were conducted by UPLC-Q-TOF-MS.

Results: The pharmacokinetic parameters of MCS have been varied, and the oral absorption effect of MCS was better than mangiferin. Also MCS had a good therapeutic effect on type 2 diabetes and NAFLD rats by regulating glucose and lipid metabolism. Sixteen potential biomarkers had been identified based on metabolomics which were related to the corresponding pathways including Pantothenate and CoA biosynthesis, fatty acid biosynthesis, citric acid cycle, arginine biosynthesis, tryptophan metabolism, etc.

Conclusions: The present study validated the favorable pharmacokinetic profiles of MCS and the biochemical mechanisms of MCS in treating type 2 diabetes and NAFLD.

Keywords: Mangiferin calcium salt, Diabetes, NAFLD, Pharmacokinetics, Metabolomics, Bioavailability

\section{Background}

Diabetes is one of the most common chronic metabolic diseases, and its incidence is gradually increasing. According to the International Diabetes Federation, the 463 million people with diabetes worldwide account for about $9.3 \%$ of the global population in 2019, of which $80 \%$ come from low- and middle-income countries. It is

\footnotetext{
* Correspondence: linhe@ccucm.edu.cn; linzhe1228@163.com

${ }^{1}$ College of Pharmacy, Changchun University of Chinese Medicine, Changchun, China

Full list of author information is available at the end of the article
}

estimated that 700 million people will account for $10.9 \%$ of the world population by 2045 [1]. Non-alcoholic fatty liver (NAFLD) is a metabolic stress liver injury, including non-alcoholic simple fatty liver, non-alcoholic steatohepatitis and related cirrhosis [2,3]. NAFLD is currently the most common liver disease in the world and the common comorbidities of diabetes. It accounts for about $75 \%$ of patients with type 2 diabetes $[4,5]$. It can cause further disorders of glucose and lipid metabolism, and gradually progress to liver fibrosis, and even cause Cirrhosis [6]. The coexistence of two diseases could affect

(c) The Author(s). 2020 Open Access This article is licensed under a Creative Commons Attribution 4.0 International License, which permits use, sharing, adaptation, distribution and reproduction in any medium or format, as long as you give appropriate credit to the original author(s) and the source, provide a link to the Creative Commons licence, and indicate if changes were made. The images or other third party material in this article are included in the article's Creative Commons licence, unless indicated otherwise in a credit line to the material. If material is not included in the article's Creative Commons licence and your intended use is not permitted by statutory regulation or exceeds the permitted use, you will need to obtain permission directly from the copyright holder. To view a copy of this licence, visit http://creativecommons.org/licenses/by/4.0/ The Creative Commons Public Domain Dedication waiver (http://creativecommons.org/publicdomain/zero/1.0/) applies to the data made available in this article, unless otherwise stated in a credit line to the data. 
the health of patients seriously [7]. Insulin resistance (IR) is currently recognized as one of the main risk factors for non-alcoholic fatty liver. It refers to the reduced sensitivity of the body to insulin, the inability to effectively synthesize and metabolize glucose. Then excessive insulin is compensatively secreted into the blood, causing hyperinsulinemia $[8,9]$. Meanwhile IR prevents insulin from efficiently inhibiting lipase activity. The increase of lipase activity will cause a large amount of adipose tissue to be broken down, and excess free fatty acids will enter the liver through the hepatic portal vein, causing fatty liver $[10,11]$. IR can also trigger oxidative stress, inflammation that promotes the deterioration of NAFLD, causing inflammation infiltration, necrosis, and even fibrosis in the liver [12, 13].

Mangiferin (2-beta-D-glucopyranosyl-1,3,6,7-tetrahydroxyxanthone, MGN) is a natural C-glucoside xanthone, which is predominantly in the fruits, leaves, and bark of Mangifera indica L. and some other medical plants including Anemarrhena asphodeloides Bge., Belamcanda chinensis (L.) DC etc. [14, 15]. It has shown many kinds of biological activities and pharmacological actions such as antioxidative, antidiabetic, hypolipidemic, antiviral, immunomodulatory, anticancer, analgesic and hepatoprotective effects [16-20]. But the characteristic of its low aqueous solubility and low fat solubility can affect the absorption process of drugs in vivo, which leads to a low bioavailability $[21,22]$. It makes us have to suffer such problems like mangiferin is hard to further develop a new medicine and its clinical application has certain limitation.

Mangiferin calcium salt (MCS) is a new salt of mangiferin which proposed to be an insulin sensitizer (Fig. 1) $[23,24]$. In the present study, the pharmacokinetic profiles of MCS in rats were evaluated to clarify the impact of single and repeated administration on its main pharmacokinetic parameters. A comparison between the major pharmacokinetic between MCS and mangiferin was subsequently executed. Metabolomics was performed with rats urine samples collected from oral administration of MCS. As our knowledge, this is the first integrated study of pharmacokinetics and metabolomics on MCS. The results of this assessment will contribute to further development of MCS as pharmaceutical products and explore the underlying mechanism of MCS in the treatment of type 2 diabetes and NAFLD.

\section{Methods}

\section{Chemicals and materials}

Mangiferin calcium salt (MCS, yellow green powder, purity: 95.25\%), Mangiferin (yellowish powder, purity: 98\%) was provided by Changzhou Deze Pharmaceutical Research Co. Ltd. (Changzhou, China). Mangiferin (purity: $98.1 \%$ ), rutin (purity: $91.9 \%$ ) as reference substance were obtained from the National Institute for the Control of Pharmaceutical and Biological Products (Beijing, China). Heparin sodium was obtained from Shanghai Huishi biochemical reagent Co., Ltd. (Shanghai, China). Acetonitrile Methanol and formic acid (HPLC grade) were obtained from Tedia Company, Inc. (Ohio, USA). Ultrapure water was produced using a Milli-Q plus (Milford, MA, USA) water purification system. Leucine enkephalin was obtained from Waters (Milford, USA). Xanthurenic acid, 5-L-Glutamyl-taurine, Citric acid, Pantothenic acid, Uric acid, Riboflavin and 3Hydroxyanthranilic acid were obtained from SigmaAldrich (St. Louis, MO, USA).

\section{Animals}

Sprague-Dawley rats (male and female, weighting 200$230 \mathrm{~g}$ ) were obtained from Changchun Yisi Laboratory Animal Technology Co., Ltd. (Changchun, China). Rats were housed with free access to food and water under standard conditions (temperature $20-24^{\circ} \mathrm{C}$, humidity 40-60\%, 12-h light/dark cycle). All experimental animals were finally euthanized by $\mathrm{CO}_{2}$ inhalation. The study

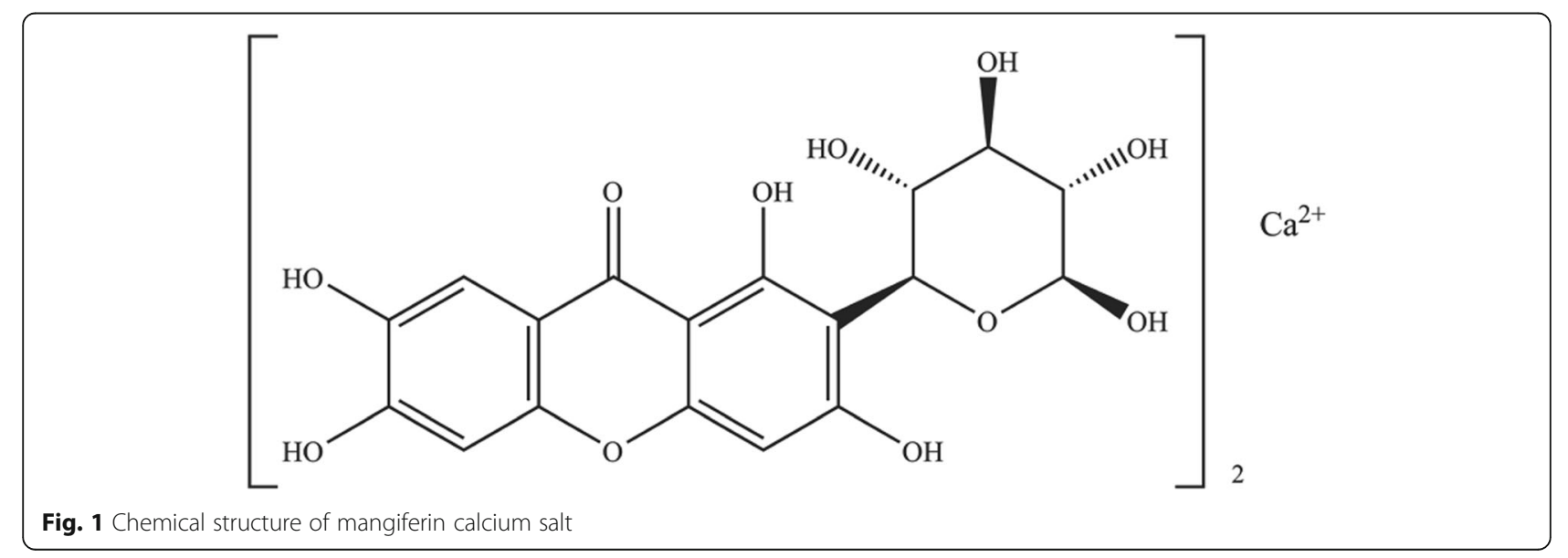


complied with the guidelines of the research commitment institution and its administrative region, as the Jilin Province Experimental Animal Management Ordinance and Changchun University of Chinese Medicine Laboratory Animal Management Measures. All experiments were approved by the Laboratory Animals Ethics Committee, Changchun University of Chinese Medicine.

\section{Administration and plasma samples collection}

MCS and mangiferin were given by gavages according to $60 \mathrm{mg} / \mathrm{kg}, 240 \mathrm{mg} / \mathrm{kg}, 960 \mathrm{mg} / \mathrm{kg}$ doses as single administration. Rats were fasted $12 \mathrm{~h}$ before the experiment and water was taken freely. In the experiment day the administration was according to the predetermined dose. Serial blood samples were collected from the orbital venous plexus $(0.3-0.5 \mathrm{~mL})$ at $0 \mathrm{~h}, 0.5 \mathrm{~h}, 1.0 \mathrm{~h}, 1.5 \mathrm{~h}, 2.0 \mathrm{~h}, 3.0 \mathrm{~h}$, $4.0 \mathrm{~h}, 6.0 \mathrm{~h}, 10.0 \mathrm{~h}, 12.0 \mathrm{~h}, 24.0 \mathrm{~h}$ after administration.

MCS and mangiferin were given by gavages according to $240 \mathrm{mg} / \mathrm{kg}$ dose, once a day for 7 days as multiple administrations. Blood samples were collected from the orbital venous plexus $(0.3-0.5 \mathrm{~mL})$ on $1,2,3,4,5,6$ days before dosing. For the last administration, Serial blood samples were collected at $0 \mathrm{~h}, 0.5 \mathrm{~h}, 1.0 \mathrm{~h}, 1.5 \mathrm{~h}, 2.0 \mathrm{~h}$, $3.0 \mathrm{~h}, 4.0 \mathrm{~h}, 6.0 \mathrm{~h}, 10.0 \mathrm{~h}, 12.0 \mathrm{~h}, 24.0 \mathrm{~h}$.

The blood samples placed in a centrifuge tube with heparin, 10,000 rpm centrifuge $10 \mathrm{~min}$. After the centrifugation, reserve the plasma in $-20^{\circ} \mathrm{C}$ refrigerator.

\section{Pharmacokinetic analysis}

The $200 \mu \mathrm{L}$ of plasma sample was placed in a $1.5 \mathrm{~mL}$ centrifuge tube, added internal standard solution $(10 \mu \mathrm{g} /$ $\mathrm{mL}$ rutin standard solution) $25 \mu \mathrm{L},(0 \mathrm{~h}$ plasma used methanol $25 \mu \mathrm{L}$ to instead), methanol $25 \mu \mathrm{L}$ (added mangiferin standard solution $25 \mu \mathrm{L}$ ), added $0.9 \mathrm{~mL}$ Acetonitrile-acetic acid (9: 1), swirl mixed 3 min, 6000 rpm centrifuged $10 \mathrm{~min}$, supernatant was dried in vacuum at $50^{\circ} \mathrm{C}$, added mobile phase $100 \mu \mathrm{L}$ to the residue, swirl mixed $2 \mathrm{~min}, 6000 \mathrm{rpm}$ centrifuged $10 \mathrm{~min}$, the supernatant was injected into High performance liquid chromatography (HPLC). Chromatographic separations were achieved using a Discovery C18 column (250*4.60 $\mathrm{mm}$ I.D, $5 \mu \mathrm{m}$, Supelco Company, USA). The mobile phase used for the separation consisted of Acetonitrile and $0.10 \%$ phosphoric acid $(25: 75, \mathrm{v} / \mathrm{v})$ delivered at $1 \mathrm{ml} /$ min flow rate. The detection wavelength was set at 318 $\mathrm{nm}$ and all measurements were performed at $30^{\circ} \mathrm{C}$.

The pharmacokinetic parameters were calculated using DAS software, and select the weighting factors to fit the atrioventricular model.

\section{Type 2 diabetes and NAFLD model construction and} administration

The SD rats were fed high-fat feed (recipe: 12\% lard, $0.5 \%$ cholate, $1 \%$ cholesterol, $5 \%$ sucrose, $81.5 \%$ basic nutritional feed). At the end of the 12th week, streptozotocin (STZ) $(30 \mathrm{mg} / \mathrm{kg})$ was intraperitoneally injected into rats to induce type 2 diabetes complicated with NAFLD model. The rats were randomly divided into the following four groups: Blank control group (BG, $n=7$ ), model control group (MG, $\mathrm{n}=7$ ) were administered with distilled water intragastrically. MCS High-dose group (MHG, n = 7), Medium dose group (MMG, $n=7$ ), Low-dose group (MLG, $\mathrm{n}=7$ ) were administered intragastrically with MCS at doses of $480 \mathrm{mg} / \mathrm{kg}, 240 \mathrm{mg} / \mathrm{kg}, 120 \mathrm{mg} / \mathrm{kg}$.

\section{Pharmacodynamics}

Blood was collected and centrifuged at $4500 \mathrm{rpm}$ low temperature centrifuge for $15 \mathrm{~min}$ to separate serum. Detect the fasting blood glucose (FBG), fasting insulin (FINS), triglyceride (TG), total cholesterol (TC), aspartate aminotransferase (AST), alanine aminotransferase (ALT) and gamma-glutamyl transpeptadase (GGT) content in rat serum. The rat liver was taken stained with hematoxylin and eosin (H\&E).

\section{Metabolomics analysis}

Urine samples were collected and centrifuged at 10,000 $\mathrm{rpm}$ for $10 \mathrm{~min}$, filtered through a $0.22 \mu \mathrm{m}$ filter membrane. Supernatant was transferred to fresh vials for ultraperformance liquid chromatography coupled with quadrupole time-of-flight mass spectrometry (UPLC-Q-TOFMS) analysis. For metabolomics analysis, the samples (each $5 \mu \mathrm{L}$ ) were injected onto a Waters ACQUITY UPLC BEH C18 Column $(1.7 \mathrm{~m}, 2.1 \mathrm{~mm} \times 50 \mathrm{~mm})$ kept at $30{ }^{\circ} \mathrm{C}$ and at a flow rate of $0.4 \mathrm{~mL} / \mathrm{min}$ using a Waters ACQUITY UPLC system coupled with a Q-TOF SYNA PT G2 High Definition Mass Spectrometer (Waters, USA). Acetonitrile (A) and $0.1 \%$ aqueous formic acid (v/v) (B) were used as gradient mobile phase. The gradient elution of A was performed as follows: $5-30 \% \mathrm{~A}$ at $0-6 \mathrm{~min}$, $30-60 \% \mathrm{~A}$ at $6-10 \mathrm{~min}, 60-100 \% \mathrm{~A}$ at $10-12 \mathrm{~min}, 100-$ $5 \% \mathrm{~A}$ at $12-12.1 \mathrm{~min}$ and then kept at $5 \% \mathrm{~A}$ for $3 \mathrm{~min}$. The positive and negative ion (ESI) modes were used in MS analysis. The source temperature was set to $120^{\circ} \mathrm{C}$. The desolvation gas temperature was set to $400^{\circ} \mathrm{C}$ and the flow was set to $800 \mathrm{~L} / \mathrm{h}$. The capillary, cone and extraction cone voltages were $3.0 \mathrm{kV}, 35 \mathrm{~V}, 5.0 \mathrm{~V}$ in positive ion mode and $2.0 \mathrm{kV}, 35 \mathrm{~V}, 5.0 \mathrm{~V}$ in negative ion mode. The full-scan mode was from 100 to $1000 \mathrm{Da}$. Accurate mass was maintained by Leucine enkephalin. MSE was applied for the MS/MS analysis with the high collision energy on $25-35 \mathrm{eV}$ and the low collision energy on $4 \mathrm{eV}$.

The quality control (QC) samples were used for method validation, which were obtained by mixing $100 \mu \mathrm{L}$ of each sample. In order to avoid errors during the entire analysis process, the QC samples were run once every 5 samples to measure the stability of the system. 
Data processing and statistical analysis

The sample was detected by UPLC-Q-TOF-MS to obtain the total ion current chromatogram of the sample. The raw data files were processed with MassLynx V4.1 and MarkerLynx Application Manager (Waters, USA) for peak detection, alignment and normalization. Multivariate analysis was performed by principal component analysis (PCA) and orthogonal projection to latent structures squares-discriminant analysis (OPLS-DA) with the EZinfo 2.0 software. All values are expressed as the mean $\pm S D$. An independent sample t-test between groups was used to evaluate the significant difference $(p<0.05)$ using SPSS statistics 13.0 software.

\section{Results}

Comparison of pharmacokinetic parameters after single administration of MCS and mangiferin

The mean plasma concentration-time curves of MCS and mangiferin in different dosage are showed in Fig. 2. The main pharmacokinetic parameters are summarized in Table 1. As be seen in Table 1, after a single
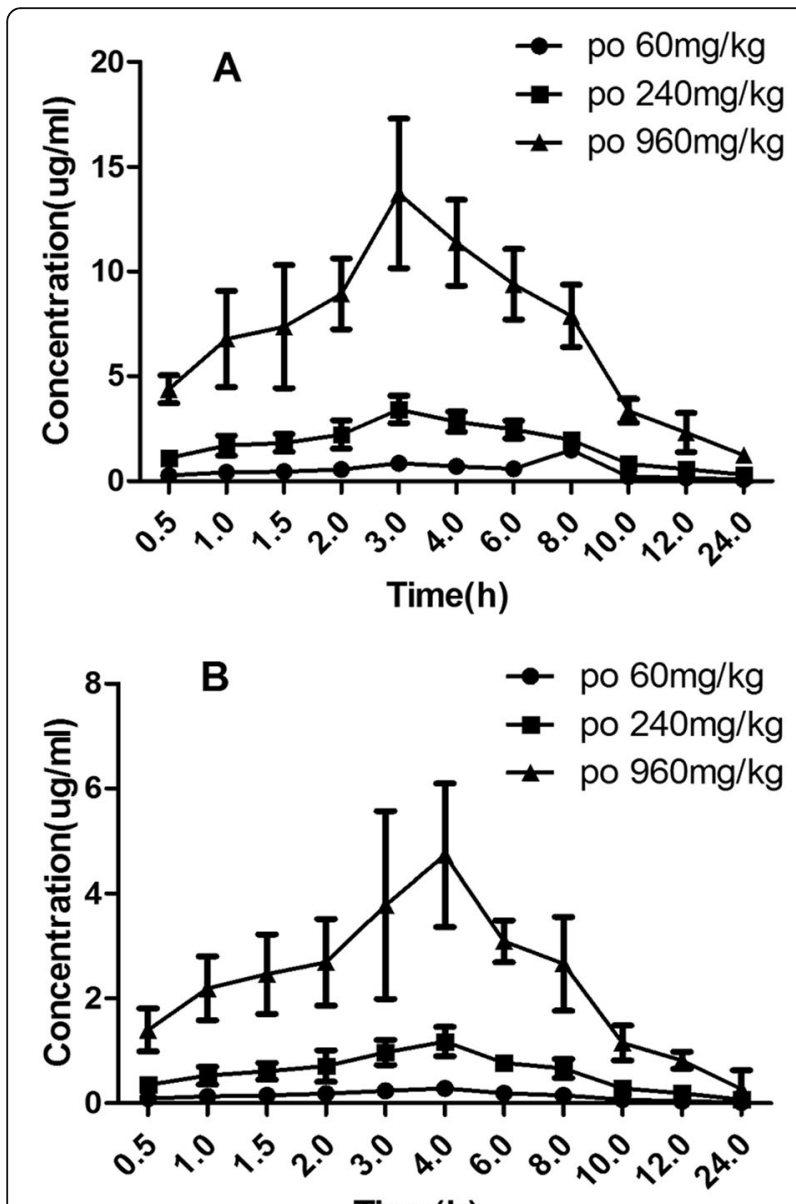

Time(h)

Fig. 2 The mean plasma concentration-time curves of MCS and mangiferin in different dosage. a MCS, b mangiferin administration of $240 \mathrm{mg} / \mathrm{kg}$, compared with $\mathrm{AUC}(0-\mathrm{t})$ $(9187.50 \mu \mathrm{g} / \mathrm{L} \cdot \mathrm{h}), \quad$ AUC $(0-\infty) \quad(9723.18 \mu \mathrm{g} / \mathrm{L} \cdot \mathrm{h}), \quad$ Tmax (4.02 h), Cmax $(1.18 \mu \mathrm{g} / \mathrm{ml})$ of mangiferin, AUC(0-t) $(28$, $126.50 \mu \mathrm{g} / \mathrm{L} \cdot \mathrm{h}), \quad$ AUC $(0-\infty) \quad(30,981.65 \mu \mathrm{g} / \mathrm{L} \cdot \mathrm{h}), \quad \mathrm{Cmax}$ $(3.42 \mu \mathrm{g} / \mathrm{ml})$ of MCS are significantly increased $(P<$ $0.05)$, Tmax $(2.99 \mathrm{~h})$ is significantly decreased $(\mathrm{P}<0.05)$. MCS has better oral absorption than mangiferin.

Comparison of pharmacokinetic parameters after multiple administration of MCS and mangiferin

The comparison of mean plasma concentration-time curves of MCS and mangiferin after multiple oral administration in dosage of $240 \mathrm{mg} / \mathrm{kg}$ are showed in Fig. 3 . The main pharmacokinetic parameters are summarized in Table 2. As be seen in Table 2, after a multiple administration of $240 \mathrm{mg} / \mathrm{kg}$, compared with AUC(0-t) $(9075.00 \mu \mathrm{g} / \mathrm{L} \cdot \mathrm{h}), \quad$ AUC $(0-\infty) \quad(9729.04 \mu \mathrm{g} / \mathrm{L} \cdot \mathrm{h}), \quad$ Tmax (4.05 h), Cmax $(1.16 \mu \mathrm{g} / \mathrm{ml})$ of mangiferin, $\mathrm{AUC}(0-\mathrm{t})(27$, $871.50 \mu \mathrm{g} / \mathrm{L} \cdot \mathrm{h}), \quad$ AUC $(0-\infty) \quad(30,789.50 \mu \mathrm{g} / \mathrm{L} \cdot \mathrm{h}), \quad$ Cmax $(3.42 \mu \mathrm{g} / \mathrm{ml})$ of MCS are significantly increased $(P<$ $0.05)$, Tmax $(3.02 \mathrm{~h})$ is significantly decreased $(\mathrm{P}<0.05)$. In addition, the main pharmacokinetic parameters of multiple and single administration of MCS have no significant difference, indicating that the absorption of MCS in rats is constant basically, and don't change with continuous administration. MCS almost has no accumulation in the body after multiple doses of administration.

\section{Pharmacodynamics study}

Type 2 diabetes patients with NAFLD often suffered from glucose and lipid metabolism disorder, and present with abnormally high fasting blood glucose, fasting insulin and HOMA-IR [25]. As our previous study (Fig. 4) [26], the serum FBG and FINS content of MG were higher than BG significantly $(P<0.01)$. Compared with MG, the level of serum FBG, FINS in MHG and MMG decreased significantly after treated with MCS $(P<0.05)$. It revealed that MCS could better improve insulin resistance. Dyslipidemia is also one of the important clinical manifestations of type 2 diabetes patients with NAFLD. Compared with BG, significant increase could be observed in serum TG, TC level in MG $(p<0.01)$. After the treatment with MCS, the concretion of serum TG, TC in MHG and MMG decreased significantly $(p<$ 0.05). It revealed that MCS could reduce the blood lipid in model rats. ALT, AST, GGT are the most significant diagnostic indicator for patients with NAFLD. Compared with BG, serum ALT and GGT activities of MG increased significantly $(p<0.01)$. After the treatment with MCS, the activities of serum ALT and GGT in MHG and MMG decreased significantly $(\mathrm{p}<0.01, \mathrm{p}<0.05)$. It revealed that MCS could improve abnormal liver function in model rats. 
Table 1 Pharmacokinetic parameters after single administration of Mangiferin calcium salt (MCS) and mangiferin in rats ( $n=6)$

\begin{tabular}{|c|c|c|c|c|c|c|}
\hline \multirow[t]{2}{*}{ Parameters } & \multicolumn{3}{|c|}{ Mangiferin calcium salt (MCS) (Mean \pm SD) } & \multicolumn{3}{|c|}{ Mangiferin (Mean \pm SD) } \\
\hline & $60 \mathrm{mg} / \mathrm{kg}$ & 240 mg/kg & 960 mg/kg & $60 \mathrm{mg} / \mathrm{kg}$ & $240 \mathrm{mg} / \mathrm{kg}$ & 960 mg/kg \\
\hline$A \cup C(0-t)(\mu \mathrm{g} / \mathrm{L} \cdot h)$ & $6988.35 \pm 1537.44$ & $28,126.50 \pm 6750.48^{*}$ & $111,771.00 \pm 32,413.59$ & $2200.00 \pm 462.89$ & $9187.50 \pm 2021.15$ & $37,077.50 \pm 11,494.23$ \\
\hline $\operatorname{AUC}(0-\infty)(\mu \mathrm{g} / L \cdot h)$ & $7714.49 \pm 2005.77$ & $30,981.65 \pm 8674.40^{*}$ & $123,314.62 \pm 38,227.53$ & $2366.16 \pm 567.88$ & $9723.18 \pm 2430.80$ & $39,101.95 \pm 12,121.60$ \\
\hline MRT(0-t)(h) & $7.28 \pm 1.67$ & $7.27 \pm 0.96$ & $7.28 \pm 0.98$ & $6.90 \pm 1.45$ & $6.89 \pm 1.58$ & $6.94 \pm 1.87$ \\
\hline $\operatorname{MRT}(0-\infty)(h)$ & $9.73 \pm 0.98$ & $9.65 \pm 0.77$ & $9.71 \pm 1.94$ & $8.69 \pm 2.17$ & $8.25 \pm 2.47$ & $8.21 \pm 1.89$ \\
\hline $\mathrm{T} 1 / 2(\mathrm{ka})(\mathrm{h})$ & $1.59 \pm 0.13$ & $1.60 \pm 0.19$ & $1.57 \pm 0.20$ & $1.66 \pm 0.22$ & $1.70 \pm 0.19$ & $1.72 \pm 0.24$ \\
\hline $\mathrm{T} 1 / 2(\mathrm{ke})(\mathrm{h})$ & $3.27 \pm 0.52$ & $3.34 \pm 0.47$ & $3.36 \pm 0.47$ & $3.15 \pm 0.41$ & $3.29 \pm 0.46$ & $3.30 \pm 0.46$ \\
\hline $\operatorname{Tmax}(h)$ & $3.11 \pm 0.25$ & $2.99 \pm 0.21^{*}$ & $3.06 \pm 0.12$ & $4.11 \pm 0.33$ & $4.02 \pm 0.28$ & $3.97 \pm 0.36$ \\
\hline $\operatorname{Cmax}(\mu \mathrm{g} / \mathrm{ml})$ & $0.86 \pm 0.21$ & $3.42 \pm 0.65^{*}$ & $13.73 \pm 3.57$ & $0.28 \pm 0.04$ & $1.18 \pm 0.28$ & $4.73 \pm 1.37$ \\
\hline V/F(c) (L/kg) & $43.50 \pm 8.27$ & $45.11 \pm 13.98^{*}$ & $45.54 \pm 14.12$ & $134.17 \pm 41.59$ & $142.41 \pm 41.30$ & $133.34 \pm 34.67$ \\
\hline CL/F(S)(L/kg·h) & $9.23 \pm 1.57$ & $9.36 \pm 2.53^{*}$ & $9.40 \pm 2.54$ & $29.55 \pm 7.68$ & $30.05 \pm 8.71$ & $28.00 \pm 7.12$ \\
\hline
\end{tabular}

Compared with mangiferin dosage of $240 \mathrm{mg} / \mathrm{kg}$ group, ${ }^{*} p<0.05$

Histological analysis showed that livers of the MG rats had lobular structures with blurred boundaries, Irregular cell cords, and hepatic sinusoidal compression became smaller or disappears, liver cells showed diffuse fat-like changes, a large number of inflammatory cells infiltration could be seen in the liver lobule, even several inflammatory necrosis merged with each other (Fig. 5).

\section{Metabolomics study}

The system of UPLC-Q-TOF-MS is used for urinary sample separation and data collection. Metabolic profiling was acquired in the ESI+ and ESI- modes. The representative based peak intensity (BPI) chromatograms in positive and negative ion modes are showed in Fig. 6a, b. PCA was performed as an unsupervised pattern recognition method to analyze the holistic metabolic variations in different groups and QCs. It can be seen from the PCA score chart (Fig. 6c, d) that the urine samples of four groups can be clearly separated in the positive ion mode $(\mathrm{R} 2 \mathrm{X}=0.679, \mathrm{Q} 2=0.410)$ and the negative ion

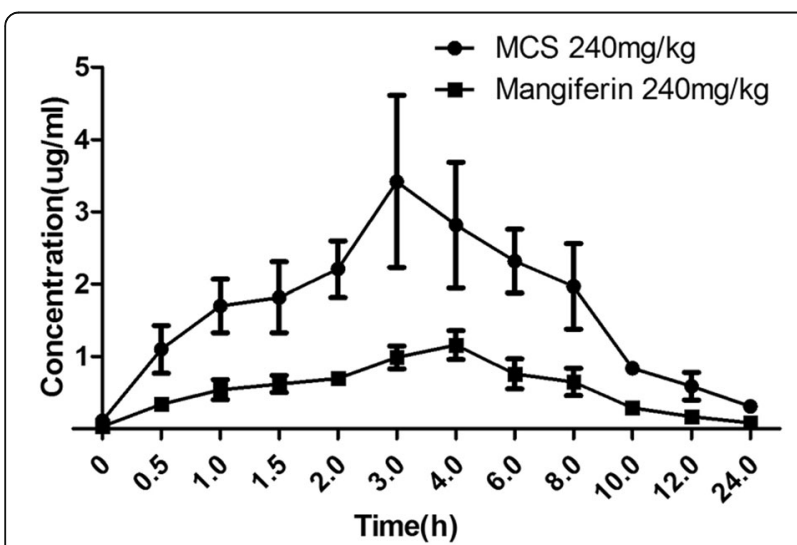

Fig. 3 The comparison of mean plasma concentration-time curves of MCS and mangiferin after multiple oral administrations in dosage of $240 \mathrm{mg} / \mathrm{kg}$ mode $(\mathrm{R} 2 \mathrm{X}=0.596, \mathrm{Q} 2=0.402)$. The $\mathrm{QC}$ samples are relatively compact in both positive ion mode and negative ion mode, revealing that the stability of the analytical system is good. BG and MG are distributed obviously in different regions, indicating that the metabolism of type 2 diabetes with NAFLD model rats has changed. MHG and MMG are close to BG which implies that the metabolic profile of MHG and MMG are returning to normal after administration of MCS.

\section{Potential biomarkers and metabolic pathway analysis}

OPLS-DA analysis was performed on the MG and MHG to find biomarkers for MCS treatment of type 2 diabetes with NAFLD. The OPLS-DA model is of good quality, and the model evaluation indexes in positive ion mode

Table 2 Pharmacokinetic parameters after multiple administration of Mangiferin calcium salt (MCS) and mangiferin in rats $(n=6)$

\begin{tabular}{lll}
\hline Parameters & Mangiferin calcium salt (MCS) & Mangiferin \\
& $240 \mathrm{mg} / \mathrm{kg}$ (Mean $\pm \mathrm{SD})$ & \\
\hline $\mathrm{AUC}(0-\mathrm{t})(\mu \mathrm{g} / \mathrm{L} \cdot \mathrm{h})$ & $27,871.50 \pm 9197.60^{*}$ & $9075.00 \pm 2631.75$ \\
$\mathrm{AUC}(0-\infty)(\mu \mathrm{g} / \mathrm{L} \cdot \mathrm{h})$ & $30,789.48 \pm 11,084.21^{*}$ & $9729.04 \pm 2724.13$ \\
MRT $(0-\mathrm{t})(\mathrm{h})$ & $7.31 \pm 1.82$ & $6.93 \pm 0.97$ \\
$\mathrm{MRT}(0-\infty)(\mathrm{h})$ & $9.77 \pm 2.40$ & $8.63 \pm 1.05$ \\
$\mathrm{~T} 1 / 2(\mathrm{ka})(\mathrm{h})$ & $0.77 \pm 0.08$ & $0.84 \pm 0.11$ \\
$\mathrm{~T} 1 / 2(\mathrm{ke})(\mathrm{h})$ & $5.54 \pm 0.72$ & $4.92 \pm 0.63$ \\
$\operatorname{Tmax}(\mathrm{h})$ & $3.02 \pm 0.09^{*}$ & $4.05 \pm 0.36$ \\
$\mathrm{Cssmin}(\mu \mathrm{g} / \mathrm{ml})$ & $0.12 \pm 0.02^{*}$ & $0.03 \pm 0.01$ \\
$\mathrm{Cmax}(\mu \mathrm{g} / \mathrm{ml})$ & $3.42 \pm 1.19^{*}$ & $1.16 \pm 0.20$ \\
$\mathrm{Cavg}(\mu \mathrm{g} / \mathrm{ml})$ & $1.16 \pm 0.09^{*}$ & $0.38 \pm 0.03$ \\
$\operatorname{V/F}(\mathrm{c})(\mathrm{L} / \mathrm{kg})$ & $75.73 \pm 18.93^{*}$ & $215.53 \pm 40.95$ \\
$\mathrm{CL} / \mathrm{F}(\mathrm{S})(\mathrm{L} / \mathrm{kg} \cdot \mathrm{h})$ & $9.48 \pm 1.52^{*}$ & $30.36 \pm 8.50$ \\
$\mathrm{FI}(\%)$ & $2.84 \pm 0.19$ & $2.97 \pm 0.21$
\end{tabular}

Compared with mangiferin group, ${ }^{*} p<0.05$ 


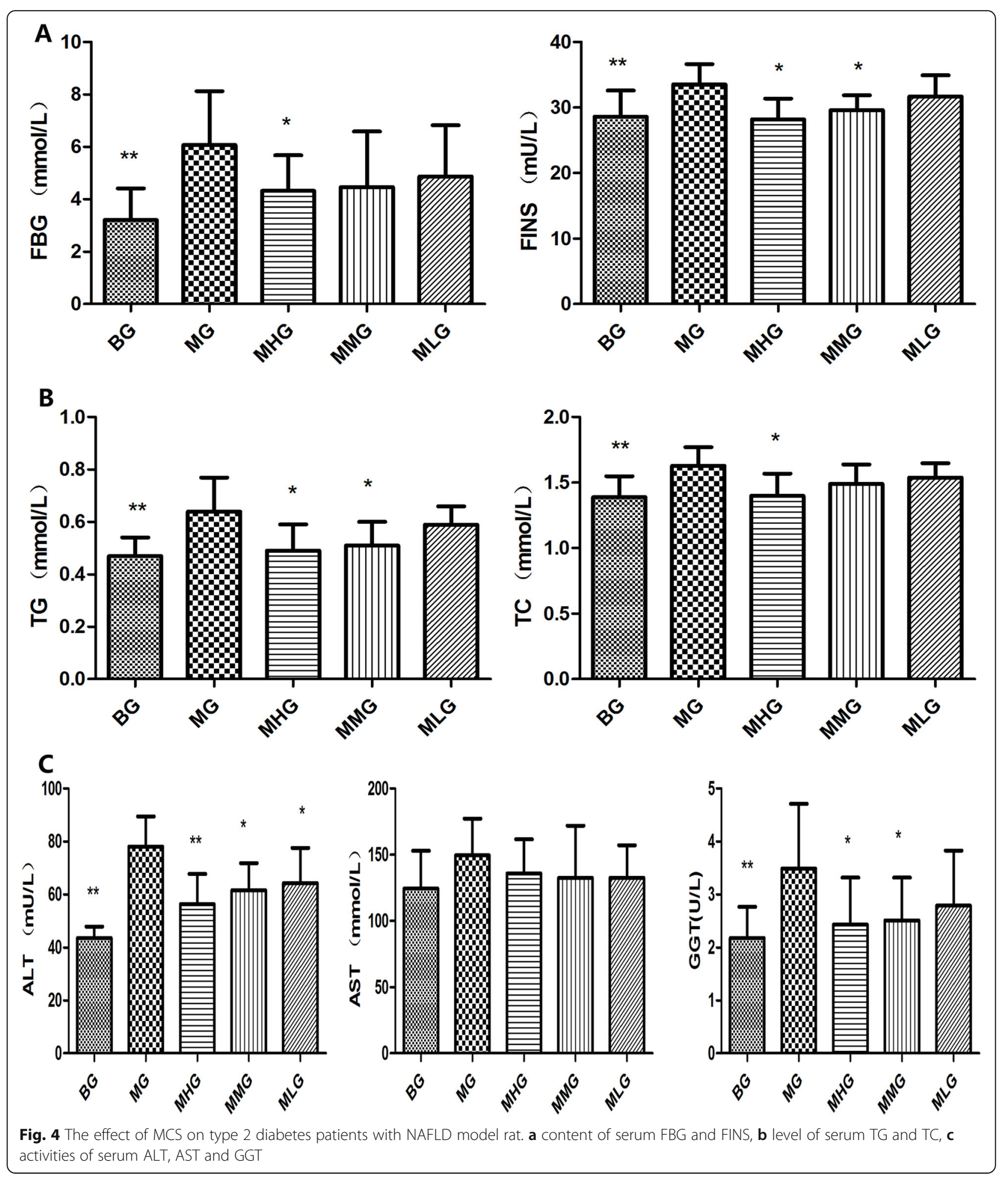

are $\mathrm{R} 2 \mathrm{Y}=0.95, \mathrm{Q} 2=0.83$, and the model evaluation indexes in negative ion mode are $\mathrm{R} 2 \mathrm{Y}=0.91, \mathrm{Q} 2=0.85$. In the OPLS-DA score chart (Fig. 7a, b), the MG and MHG can be clearly divided into two parts, indicating that the difference between the groups is much larger than the difference between the groups. In S-plot (Fig. 7c, d), the points at both ends of the S-type are potential biomarkers, and the VIP $>1.0$ and $p$-value $<0.05$ between DG, MG and MHG are used as the criterion for another biomarker. Finally, 16 endogenous metabolites were 


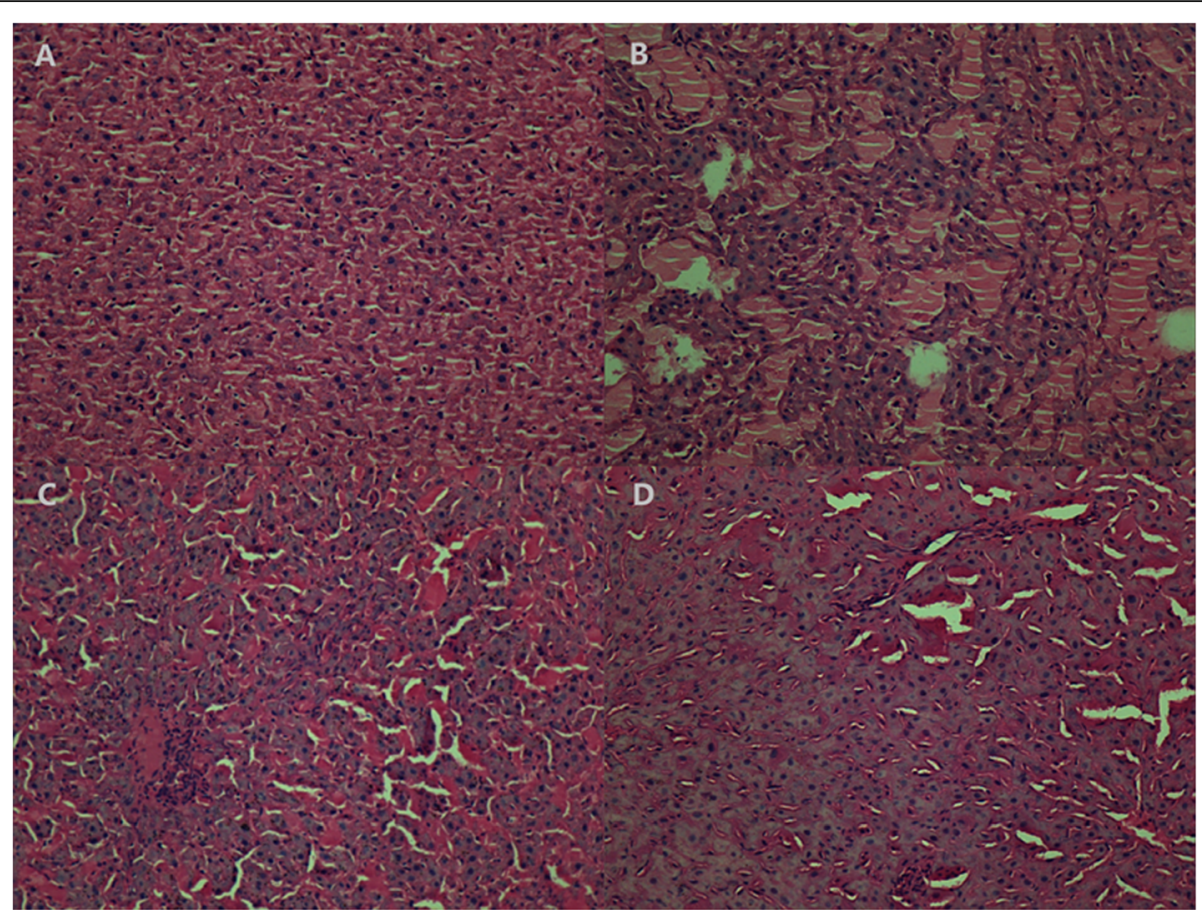

Fig. 5 The histological examination of liver tissue (magnification×200). The data are representative H\&E stained sections from each group. a Blank control group, BG, b model control group, MG, c MCS High-dose group, MHG, d Medium dose group, MMG

identified as potential biomarkers (Table 3). Metabolic pathways affected by the biomarkers can be obtained by MetPA (http://metpa.metabolomics.ca/) analysis, including Taurine and hypotaurine metabolism, Pantothenate and $\mathrm{CoA}$ biosynthesis, Alanine, aspartate and glutamate metabolism, Riboflavin metabolism, Arginine biosynthesis, Citrate cycle (TCA cycle), Glyoxylate and dicarboxylate metabolism, Tryptophan metabolism, Primary bile acid biosynthesis, Fatty acid biosynthesis and Purine metabolism (Fig. 8a). Searching these metabolic
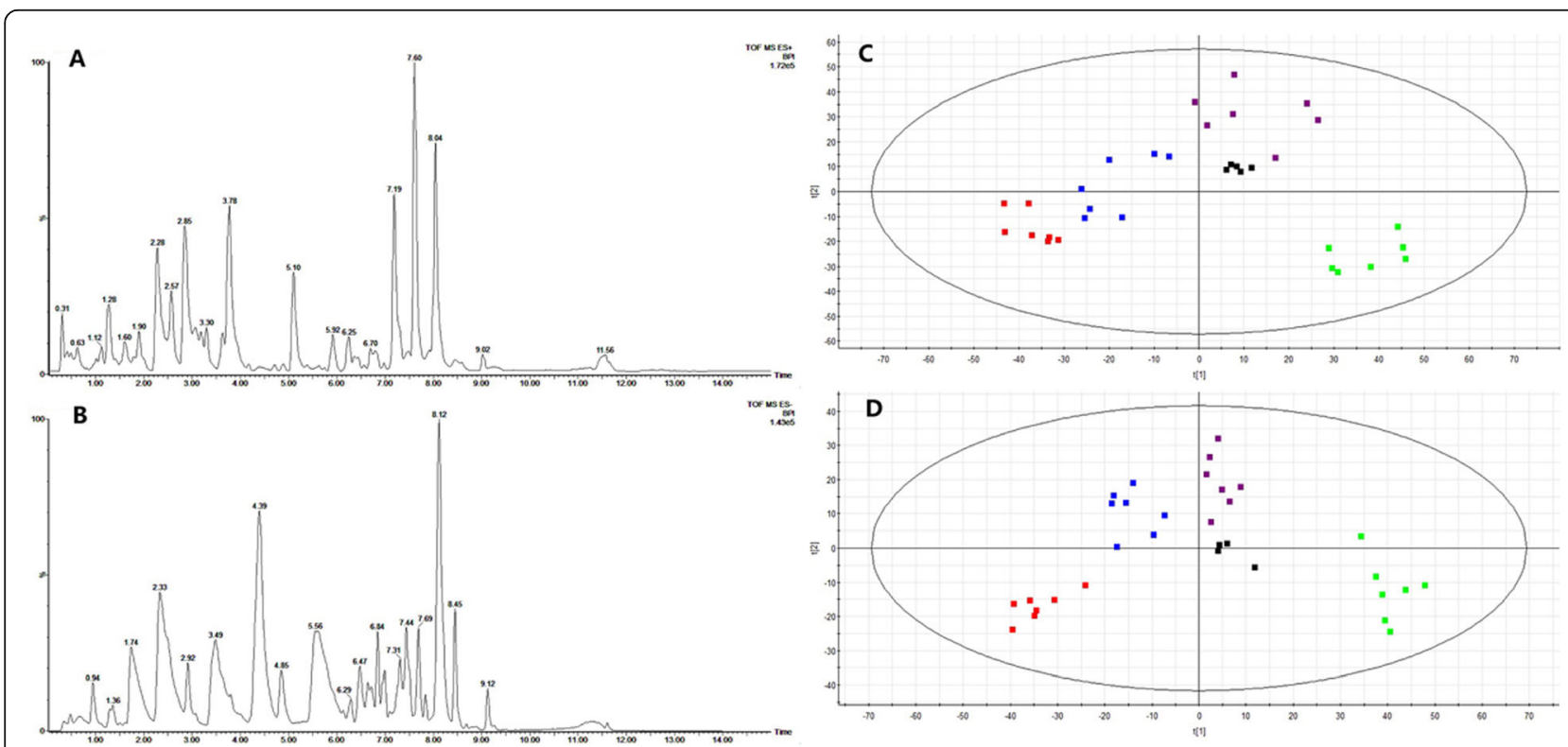

Fig. 6 PCA score plots of urine metabolic profiling of BG (red), MG (green), MHG (blue), MMG (violet) and QCs (black) in positive mode (a) and negative mode $(\mathbf{b})$ 

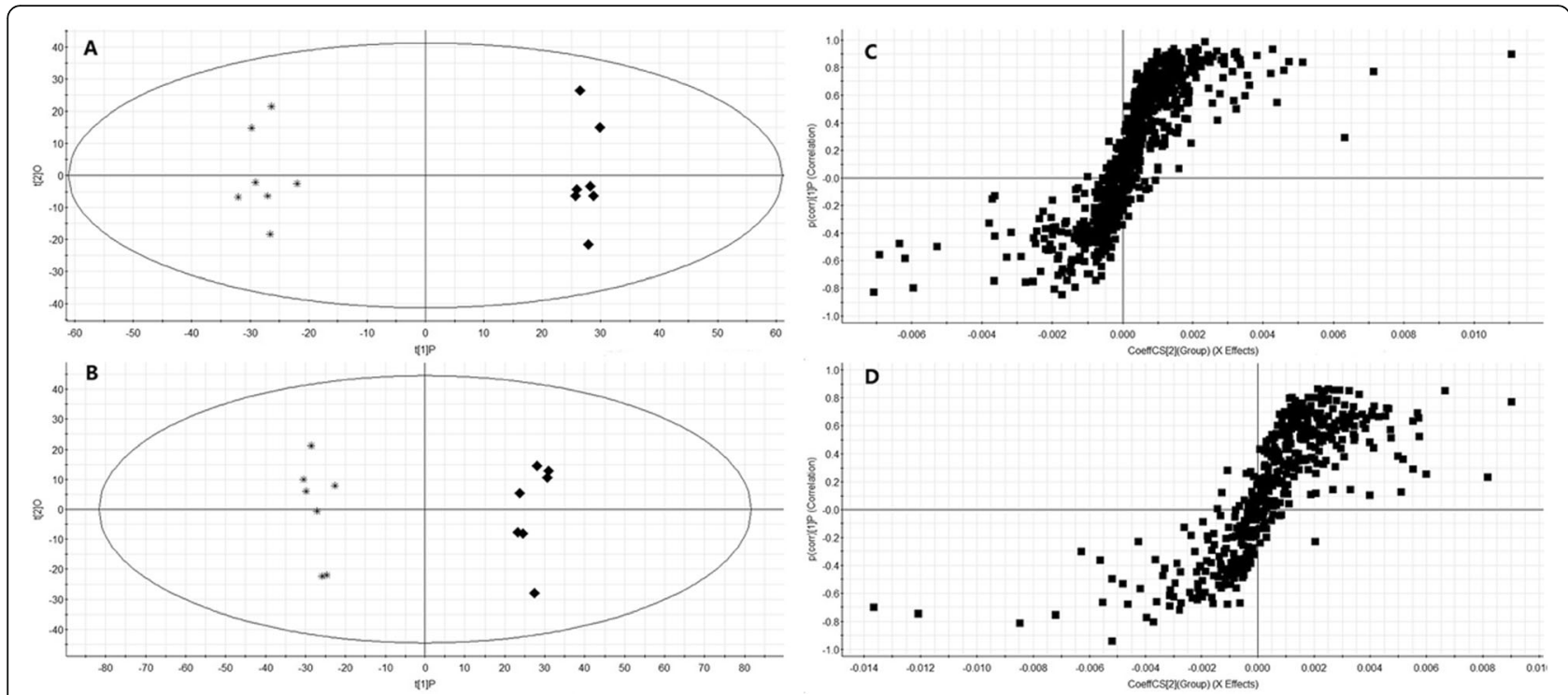

Fig. 7 OPLS-DA score plots of urine metabolic profiling of MG (-a) and MHG (*) in positive mode (a) and negative mode (b) and OPLS-DA S-plots in positive mode $(\mathbf{c})$ and negative mode $(\mathbf{d})$

pathways and biomarkers in KEGG database and establishing the metabolic correlation network and heatmap of metabolites affected by MCS treatment (Fig. 8b, c).

\section{Discussion}

Mangiferin is widely found in many edible and medicinal plants and has many pharmacological activities, such as antitussive, expectorant, antiasthmatic, central depression, anti-diabetic, antioxidant, anti-inflammatory, bacteriostatic, anti-viral, anti-tumor, choleretic and immunomodulatory, so it has attracted the attention of researchers $[14,19]$. Especially it has a good improvement effect on metabolic diseases such as diabetes, nonalcoholic fatty liver and hyperuricemia [27]. It has been reported that mangiferin under hypoxic conditions can promote the absorption of glucose by cells and improve insulin resistance and damage in fat cells [28]. It can significantly reduce blood glucose levels, increase glucose

Table $\mathbf{3}$ Identification results of potential biomarkers

\begin{tabular}{|c|c|c|c|c|c|c|c|}
\hline Mode & RT & Measured mass & VIP & Formula & Error (ppm) & Identification & Trenda \\
\hline \multirow[t]{9}{*}{$\overline{\mathrm{ESI}}+$} & 7.19 & 206.0438 & 4.78 & $\mathrm{C} 10 \mathrm{H} 7 \mathrm{NO} 4$ & 1.0 & Xanthurenic acid & up \\
\hline & 2.59 & 338.1134 & 3.07 & $\mathrm{C} 7 \mathrm{H} 14 \mathrm{~N} 2 \mathrm{O} 6 \mathrm{~S}$ & 3.0 & 5-L-Glutamyl-taurine & up \\
\hline & 2.30 & 105.0414 & 2.91 & $\mathrm{C} 10 \mathrm{H} 18 \mathrm{~N} 4 \mathrm{O} 6$ & -6.7 & Argininosuccinic acid & down \\
\hline & 1.18 & 220.1181 & 2.86 & $\mathrm{C} 9 \mathrm{H} 17 \mathrm{NO} 5$ & 0.9 & Pantothenic acid & up \\
\hline & 1.93 & 162.0673 & 2.80 & $\mathrm{C} 12 \mathrm{H} 22 \mathrm{~N} 2 \mathrm{O} 6 \mathrm{~S}$ & 0.6 & D-Pantothenoyl-L-cysteine & down \\
\hline & 0.51 & 191.0206 & 2.18 & $\mathrm{C} 6 \mathrm{H} 8 \mathrm{O} 7$ & -2.0 & Citric acid & up \\
\hline & 3.08 & 164.0711 & 2.13 & $\mathrm{C9H} 9 \mathrm{NO} 2$ & -3.0 & 3-Methyldioxyindole & up \\
\hline & 8.04 & 426.3567 & 1.77 & $\mathrm{C} 25 \mathrm{H} 47 \mathrm{NO} 4$ & 2.6 & Vaccenyl carnitine & up \\
\hline & 1.70 & 135.0641 & 1.70 & $\mathrm{C} 5 \mathrm{H} 1004$ & -8.1 & 2,3-Dihydroxyvaleric acid & down \\
\hline \multirow[t]{7}{*}{ ESI- } & 2.74 & 143.1067 & 6.68 & $\mathrm{C} 8 \mathrm{H} 16 \mathrm{O} 2$ & -7.7 & Caprylic acid & up \\
\hline & 0.48 & 167.0201 & 3.11 & $\mathrm{C} 5 \mathrm{H} 4 \mathrm{~N} 4 \mathrm{O} 3$ & 6.0 & Uric acid & down \\
\hline & 6.37 & 377.1454 & 2.79 & $\mathrm{C} 17 \mathrm{H} 20 \mathrm{~N} 4 \mathrm{O} 6$ & 0.8 & Riboflavin & up \\
\hline & 3.30 & 173.0808 & 2.32 & $\mathrm{C} 8 \mathrm{H} 14 \mathrm{O} 4$ & -6.4 & Suberic acid & down \\
\hline & 0.40 & 124.0067 & 2.29 & $\mathrm{C} 2 \mathrm{H} 7 \mathrm{NO} 3 \mathrm{~S}$ & 6.4 & Taurine & up \\
\hline & 1.13 & 154.0505 & 1.53 & $\mathrm{C} 7 \mathrm{H} 7 \mathrm{NO} 3$ & 2.6 & 3-Hydroxyanthranilic acid & up \\
\hline & 2.24 & 157.0882 & 1.28 & $\mathrm{C} 8 \mathrm{H} 14 \mathrm{O} 3$ & 1.9 & 3-Oxooctanoic acid & down \\
\hline
\end{tabular}



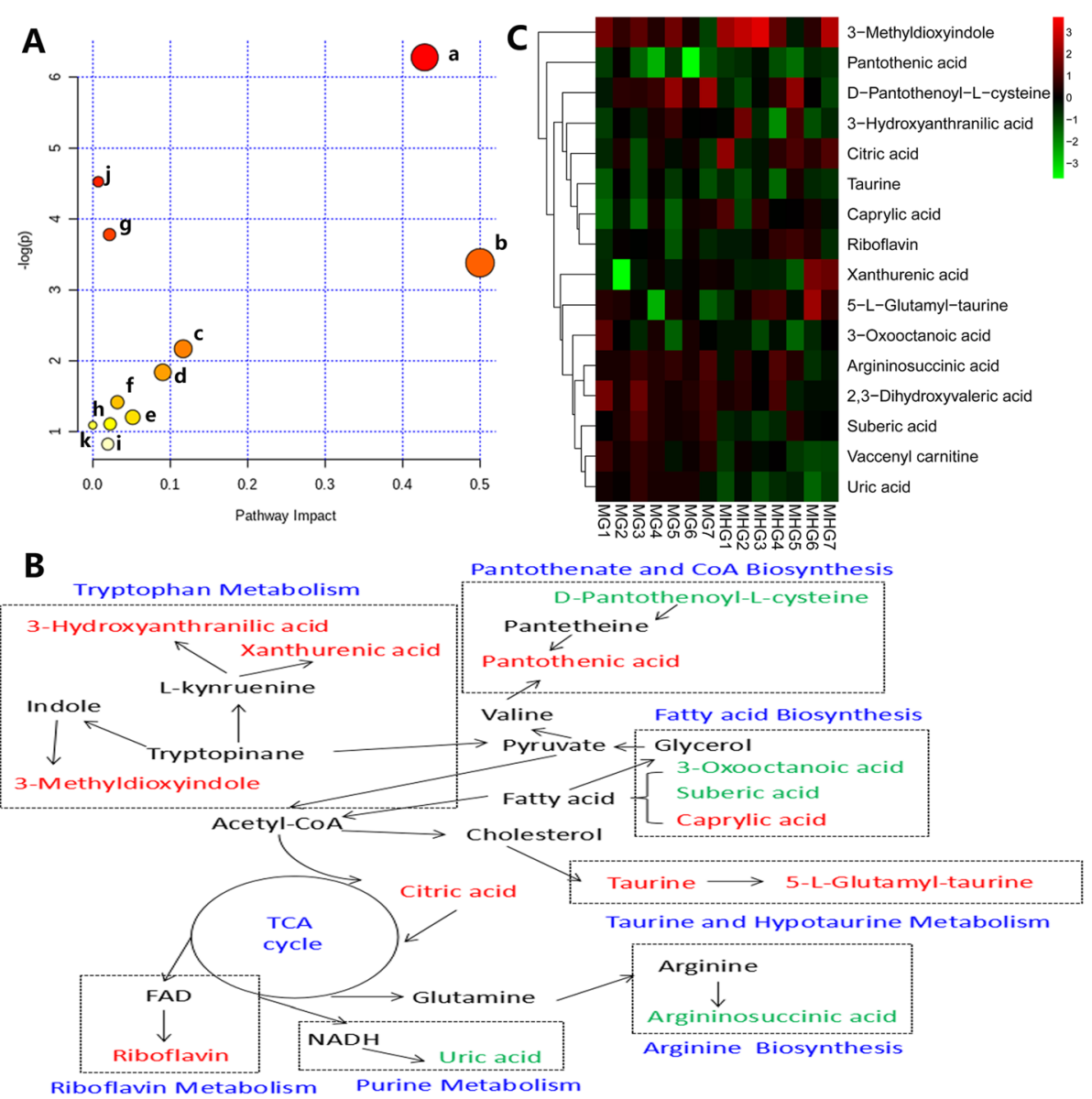

Fig. 8 Correlation networks of potential biomarkers and heatmap of metabolites responding to MCS. a Metabolic pathway enrichment analysis (from a to $\mathrm{k}$ are Taurine and hypotaurine metabolism, Pantothenate and CoA biosynthesis, Alanine, aspartate and glutamate metabolism, Riboflavin metabolism, Arginine biosynthesis, Citrate cycle (TCA cycle), Glyoxylate and dicarboxylate metabolism, Tryptophan metabolism, Primary bile acid biosynthesis, Fatty acid biosynthesis and Purine metabolism), b Metabolic pathway networks analysis (the red color indicates upregulated level; the green color indicates down-regulated level), c Heatmap of metabolites

tolerance, increase serum insulin levels, and promote islet regeneration and $\beta$ cell proliferation, and inhibit $\beta$ cell apoptosis $[29,30]$. In addition, mangiferin can reduce insulin resistance by regulating the redistribution of sarcolemma and intracellular fatty acid transfer enzymes in skeletal muscle [31]. It still can inhibit liver diacylglycerol acyltransferase gene expression, reduce liver quality and liver TG and TC levels, and inhibit excessive accumulation of lipid in the liver [32]. Although mangiferin has many pharmacological effects, due to its low solubility, it cannot be completely dissolved in the aqueous phase and the oil phase, has poor oral absorption, and has low bioavailability, which limits further clinical development and application [33]. At present, the modification of mangiferin derivatives and their metabolic active products may be an important direction for in-depth research and clinical application for it [34]. Mangiferin calcium salt (MCS) is a derivative of mangiferin, which may be an effective way to solving the above problems.
Therefore, we detected the blood drug concentration of MCS and mangiferin in single and multiple doses, and calculated their pharmacokinetic parameters in different time. Compared with mangiferin, the Tmax of MCS was advanced, and the AUC, Cmax of MCS increased significantly indicating that the degree of oral absorption of MCS was improved.

Shorter peak time showed that the rate of absorption of MCS was faster than the monomer of mangiferin. Moreover MCS has higher bioavailability than mangiferin. Compared the pharmacokinetic parameters between single and multiple dose oral administration of MCS, MRT and T1/2 had no significant change, which indicated that the absorption of MCS in rats is basically constant, and it will not change with continuous administration. These results showed that compared to mangiferin, MCS had a faster absorption rate, better absorption degree and its absorption was more constant. 
IR plays a very key role in the pathogenesis of type 2 diabetes and NAFLD [35]. IR causes the body to produce compensation and secrete more insulin due to the body's decreased glucose regulation function. This result leads to the hydrolysis of triglycerides in the body and the increase of plasma fatty acid content, which ultimately promotes the increase of blood sugar and is excreted from the kidney [36, 37]. At the same time, IR prevents insulin from efficiently inhibiting lipase activity. The increase in this enzyme activity will cause a large amount of fat to be broken down and enter the liver through the hepatic portal vein, causing simple fatty liver, which is related to oxidative stress Lipid peroxidation and the further action of inflammatory factors will lead to increased triglyceride content and destroy liver function [38-40]. Our previous research results show that MCS can significantly reduce fasting blood glucose and fasting insulin levels in rats with type 2 diabetes and NAFLD, reduce serum lipid levels, improve liver function, repair liver damage, and significantly increase the antioxidant capacity of model rats Ability to reduce oxidative stress and lipid peroxidation damage in model rats. It reveals that MCS has a certain therapeutic effect on type 2 diabetes and NAFLD. Moreover, 16 potential biomarkers related to type 2 diabetes and NAFLD were changed in the urine of MCS treated rats in our metabolomic study.

Among these metabolites, D-Pantothenoyl-L-cysteine is involved in the biosynthetic pathway of pantothenic acid and CoA, and is a synthetic precursor of Pantothenic acid that is a water-soluble vitamin required for life support. It is involved in the synthesis of acetyl-CoA and plays an important role in the metabolism of protein, fat, and sugar in the body [41]. Riboflavin is a prosthetic group of flavinases in the electron transfer process of the respiratory chain, which has anti-lipid peroxidation effect. As an important oxidoreductase in the body, flavinase participates in sugar oxidation metabolism and promotes the conversion of pyruvate to acetyl-CoA Process, thereby improving energy supply [42]. The average content of riboflavin in the urine of type 2 diabetes patients is generally lower than that of the normal population [43]. Caprylic acid, suberic acid and 3-oxooctanoic acid are important unsaturated fatty acids in the body. They regulate metabolism and cell signal transduction in the body, participate in the synthesis, decomposition and metabolism of fatty acids, and are converted into acetylCoA through beta oxidation into the citric acid cycle $[44,45]$. Vaccenyl carnitine is a long-chain acyl fatty acid derivative of carnitine. Mitochondrial carnitine palmitoyl transferase II deficiency patients accumulate long-chain acyl fatty acid derivatives in the cytoplasm and serum [46]. It is a normal recessive disease of fatty acid metabolism. Abnormal oxidation of mitochondrial fatty acids can lead to hypoglycemia, liver dysfunction, myopathy, cardiomyopathy and encephalopathy $[47,48]$. Argininosuccinic acid is a metabolite in the main biochemical pathway of lysine. It is an intermediate for the metabolism of lysine and sucralose. Studies in rats have shown that the level of argininosuccinic acid increases in prediabetes, so aminoadipate can be used as a predictive biomarker for the development of diabetes [49].

Xanthurenic acid, 3-Methyldioxyindole, 3Hydroxyanthranilic acid are metabolite of tryptophan metabolism. Tryptophan and its metabolites play an important role in various physiological processes in the body, which mainly affect the immune system and nervous system. It is closely related to various diseases such as autoimmune diseases, abnormal liver function, CNS diseases and cancer [50]. 5-LGlutamyl taurine is an intermediate of taurine metabolism. Taurine has many biological functions, such as cell membrane stabilizers and ion transmission accelerators, which can affect body fat metabolism, reduce inflammation and oxidative stress. Uric acid is a product of purine metabolism [51]. Abnormal purine metabolism can cause uric acid accumulation in the body, leading to gout, chronic kidney disease, diabetes, hyperlipidemia, hypertension and other diseases [52].

These metabolites are closely related to the occurrence and development of type 2 diabetes and NAFLD. In this study, MCS can exert its therapeutic effect by regulating the above metabolites.

\section{Conclusions}

In summary, our results showed that the pharmacokinetic profiles of MCS were better than mangiferin. Also MCS had a good therapeutic effect on type 2 diabetes with NAFLD rats by regulating glycolipid metabolism. The metabolomics could provide effective information for metabolic changes in model rats after administration of MCS in urine. However the animal models do not fully reflect human NAFLD, and there are still some debates about the occurrence of NAFLD in T2DM. Our results might help to provide useful evidence for mechanism and clinical applications of MCS acting on type 2 diabetes and NAFLD.

\footnotetext{
Abbreviations

MCS: Mangiferin calcium salt; NAFLD: Non-alcoholic fatty liver; IR: Insulin resistance; MGN: Mangiferin; HPLC: High performance liquid chromatography; STZ: Streptozotocin; BG: Blank control group; MG: Model control group; MHG: MCS High-dose group; MMG: Medium dose group; MLG: Low-dose group; FBG: Fasting blood glucose; FINS: Fasting insulin; TG: Triglyceride; TC: Total cholesterol; AST: Aspartate aminotransferase; ALT: alanine aminotransferase; GGT: gamma-glutamyl transpeptadase; H\&E: Hematoxylin and eosin; UPLC-Q-TOF-MS: Ultra-performance liquid chromatography coupled with quadrupole time-of-flight mass spectrometry; QC: Quality control; PCA: Principal component analysis; OPLS-DA: Orthogonal projection to latent structures squares-discriminant analysis
} 


\section{Acknowledgments}

Not applicable.

\section{Authors' contributions}

Research design: $\mathrm{XH}$ and ZL; research implementation: WY, GL and HL; data analysis: WW, WY and $\mathrm{HT}$; writing —original draft preparation: $\mathrm{HL}_{\text {; }}$

writing - review and editing: YL and HL. All authors read and approved the final manuscript.

\section{Funding}

This research was funded by the Jilin Province Jilin Province Industrial Technology Project (grant number 2013C017-2 and 20200032-2), Jilin Province Science and Technology Development Project (grant number $20200801028 \mathrm{GH}$ ). The funding body had no role in the design of this study and collection, analysis, and interpretation of the data, as well as in the preparation of the manuscript.

\section{Availability of data and materials}

The datasets used and/or analyzed during the current study are available from the corresponding author on reasonable request.

\section{Ethics approval and consent to participate}

The study complied with the guidelines of the research commitment institution and its administrative region, as the Jilin Province Experimental Animal Management Ordinance and Changchun University of Chinese Medicine Laboratory Animal Management Measures. All experiments were approved by the Laboratory Animals Ethics Committee, Changchun University of Chinese Medicine.

\section{Consent for publication}

Not applicable.

\section{Competing interests}

The authors declare no conflict of interest.

\section{Author details}

${ }^{1}$ College of Pharmacy, Changchun University of Chinese Medicine, Changchun, China. ${ }^{2}$ Changzhou Deze Drug Research Co., Ltd, Changzhou, China.

Received: 20 June 2020 Accepted: 27 July 2020

\section{Published online: 06 August 2020}

\section{References}

1. Pinchevsky $Y$, et al. Demographic and clinical factors associated with development of type 2 diabetes: a review of the literature. Int J Gen Med. 2020;13:121-9.

2. Kumar R, Priyadarshi RN, Anand U. Non-alcoholic fatty liver disease: growing burden, adverse outcomes and associations. J Clin Transl Hepatol. 2020;8(1): 76-86.

3. Ke W, et al. Dietary Platycodon grandiflorus Attenuates Hepatic Insulin Resistance and Oxidative Stress in High-Fat-Diet Induced Non-Alcoholic Fatty Liver Disease. Nutrients. 2020;12(2):480.

4. Tuong TTK, et al. Non-Alcoholic Fatty Liver Disease in Patients with Type 2 Diabetes: Evaluation of Hepatic Fibrosis and Steatosis Using Fibroscan. Diagnostics (Basel). 2020;10(3):159

5. Matsuzaka T, Shimano H. A new perspective on type 2 diabetes, dyslipidemia, and non-alcoholic fatty liver disease. J Diabetes Investig. 2020; 11(3):533-4.

6. Saponaro C, Gaggini M, Gastaldelli A. Nonalcoholic fatty liver disease and type 2 diabetes: common pathophysiologic mechanisms. Curr Diab Rep. 2015;15(6):607.

7. Cusi K. Treatment of patients with type 2 diabetes and non-alcoholic fatty liver disease: current approaches and future directions. Diabetologia. 2016 59(6):1112-20

8. Tao $Z$, et al. High-fat diet from perilla oil induces insulin resistance despite lower serum lipids and increases hepatic fatty acid oxidation in rats. Lipids Health Dis. 2014:13:15.

9. Byeon SK, et al. High-throughput and rapid quantification of lipids by nanoflow UPLC-ESI-MS/MS: application to the hepatic lipids of rabbits with nonalcoholic fatty liver disease. Anal Bioanal Chem. 2016;408(18):4975-85.
10. Sun R, et al. Silybin ameliorates hepatic lipid accumulation and modulates global metabolism in an NAFLD mouse model. Biomed Pharmacother. 2020;123:109721.

11. Soder J, et al. Indication of metabolic inflexibility to food intake in spontaneously overweight Labrador retriever dogs. BMC Vet Res. 2019;15(1): 96.

12. Dhibi M, et al. The intake of high fat diet with different trans fatty acid levels differentially induces oxidative stress and non alcoholic fatty liver disease (NAFLD) in rats. Nutr Metab. 2011;8:65.

13. Chai SY, et al. Differential patterns of insulin secretion and sensitivity in patients with type 2 diabetes mellitus and nonalcoholic fatty liver disease versus patients with type 2 diabetes mellitus alone. Lipids Health Dis. 2014; 13:7.

14. Imran M, et al. Mangiferin: a natural miracle bioactive compound against lifestyle related disorders. Lipids Health Dis. 2017;16(1):84.

15. Rasool M, et al. Mangiferin, a natural polyphenol protects the hepatic damage in mice caused by CCl4 intoxication. Comp Clin Pathol. 2011;21(5): 865-72.

16. Marquez $L$, et al. Mangiferin decreases inflammation and oxidative damage in rat brain after stress. Eur J Nutr. 2012;51(6):729-39.

17. Li HW, et al. Protective effects of mangiferin in subchronic developmental lead-exposed rats. Biol Trace Elem Res. 2013;152(2):233-42.

18. Liu YW, et al. Suppression of methylglyoxal hyperactivity by mangiferin can prevent diabetes-associated cognitive decline in rats. Psychopharmacology. 2013;228(4):585-94.

19. Du S, et al. Mangiferin: an effective therapeutic agent against several disorders (review). Mol Med Rep. 2018;18(6):4775-86.

20. Szandruk M, Merwid-Lad A, Szelag A. The impact of mangiferin from Belamcanda chinensis on experimental colitis in rats. Inflammopharmacology. 2018;26(2):571-81.

21. Kammalla AK, et al. Comparative pharmacokinetic study of mangiferin after oral administration of pure mangiferin and US patented polyherbal formulation to rats. AAPS PharmSciTech. 2015;16(2):250-8.

22. Liu M, et al. Solubility, Antioxidation, and Oral Bioavailability Improvement of Mangiferin Microparticles Prepared Using the Supercritical Antisolvent Method. Pharmaceutics. 2020;12:2

23. Xu G; Hainan Deze Pharmaceutical Research Co., Ltd. Mangiferin Salt and Method of Preparing the Same and Use Thereof. CN101108869B; 2008.

24. Telang $\mathrm{M}$, et al. Therapeutic and cosmetic applications of mangiferin: a patent review. Expert Opin Ther Patents. 2013;23(12):1561-80.

25. Nar A, Gedik O. The effect of metformin on leptin in obese patients with type 2 diabetes mellitus and nonalcoholic fatty liver disease. Acta Diabetol. 2009;46(2):113-8.

26. LIN H, et al. Effect of Mangiferin calcium salt on type 2 diabetes mellitus and nonalcoholic fatty liver in rats. Chinese J Clin Pharmacol. 2018;34(13): 1543-6.

27. Apontes $P$, et al. Mangiferin stimulates carbohydrate oxidation and protects against metabolic disorders induced by high-fat diets. Diabetes. 2014;63(11): 3626-36.

28. YANG C-Q, et al. Mangiferin ameliorates insulin resistance by inhibiting inflammation and regulatiing adipokine expression in adipocytes under hypoxic condition. Chin J Nat Med. 2017;15(9):0664-73.

29. Costes S. Targeting protein misfolding to protect pancreatic beta-cells in type 2 diabetes. Curr Opin Pharmacol. 2018:43:104-10.

30. Wang $\mathrm{HL}$, et al. Mangiferin facilitates islet regeneration and beta-cel proliferation through upregulation of cell cycle and beta-cell regeneration regulators. Int J Mol Sci. 2014;15(5):9016-35.

31. Zhou $\mathrm{L}$, et al. Mitigation of insulin resistance by Mangiferin in a rat model of fructose-induced metabolic syndrome is associated with modulation of CD36 redistribution in the skeletal muscle. J Pharmacol Exp Ther. 2016; 356(1):74-84.

32. Xing $X$, et al. Mangiferin treatment inhibits hepatic expression of acylcoenzyme a:diacylglycerol acyltransferase-2 in fructose-fed spontaneously hypertensive rats: a link to amelioration of fatty liver. Toxicol Appl Pharmacol. 2014;280(2):207-15.

33. Khurana RK, et al. Improving the biopharmaceutical attributes of mangiferin using vitamin E-TPGS co-loaded self-assembled phosholipidic nano-mixed micellar systems. Drug Deliv Transl Res. 2018;8(3):617-32.

34. Quadri F, Telang M, Mandhare A. Therapeutic and cosmetic applications of mangiferin: an updated patent review (patents published after 2013). Expert Opin Ther Pat. 2019;29(6):463-79. 
35. Na LX, et al. Curcumin improves insulin resistance in skeletal muscle of rats. Nutr Metab Cardiovasc Dis. 2011;21(7):526-33.

36. Zhang $Q$, et al. Mangiferin improved Palmitate-induced-insulin resistance by promoting free fatty acid metabolism in HepG2 and $\mathrm{C} 2 \mathrm{C} 12$ cells via PPARalpha: Mangiferin improved insulin resistance. J Diabetes Res. 2019; 2019:2052675.

37. Wang C, et al. The compound of Mangiferin-Berberine salt has potent activities in modulating lipid and glucose metabolisms in HepG2 cells. Biomed Res Int. 2016;2016:8753436.

38. Wang $\mathrm{H}$, et al. Mangiferin ameliorates fatty liver via modulation of autophagy and inflammation in high-fat-diet induced mice. Biomed Pharmacother. 2017;96:328-35.

39. Li J, et al. Mangiferin improves hepatic lipid metabolism mainly through its metabolite-Norathyriol by modulating SIRT-1/AMPK/SREBP-1c signaling. Front Pharmacol. 2018;9:201.

40. Stanford Kl, Goodyear LJ. Exercise and type 2 diabetes: molecular mechanisms regulating glucose uptake in skeletal muscle. Adv Physiol Educ. 2014;38(4):308-14

41. Oxenkrug G. Insulin resistance and Dysregulation of tryptophan-Kynurenine and Kynurenine-Nicotinamide adenine dinucleotide metabolic pathways. Mol Neurobiol. 2013;48(2):294-301.

42. Mazur-Bialy Al, Pochec E. Riboflavin Reduces Pro-Inflammatory Activation of Adipocyte-Macrophage Co-culture. Potential Application of Vitamin B2 Enrichment for Attenuation of Insulin Resistance and Metabolic Syndrome Development. Molecules. 2016;21:12.

43. Zhou SS, et al. B-vitamin consumption and the prevalence of diabetes and obesity among the US adults: population based ecological study. BMC Public Health. 2010;10:746.

44. ARUOMA Ol, et al. The antioxidant action of taurine, hypotaurine and their metabolic precursors. Biochem J. 1988;256:251-5.

45. Casquel De Tomasi L, et al. Pathological hypertrophy and cardiac dysfunction are linked to aberrant endogenous unsaturated fatty acid metabolism. PLoS One. 2018;13(3):e0193553.

46. GEMPEL K, et al. Screening for carnitine palmitoyltransferase II deficiency by tandem mass spectrometry. J Inherit Metab Dis. 2002;25:17-27.

47. SHEN JJ, et al. Acylcarnitines in fibroblasts of patients with long-chain 3hydroxyacyl-CoA dehydrogenase deficiency and other fatty acid oxidation disorders. J Inherit Metab Dis. 2000;23:27-44.

48. Spiekerkoetter $\mathrm{U}$, et al. Changes in blood carnitine and acylcarnitine profiles of very long-chain acyl-CoA dehydrogenase-deficient mice subjected to stress. Eur J Clin Investig. 2004;34:191-6.

49. Yuan $W$, et al. Amine metabolomics of hyperglycemic endothelial cells using capillary LC-MS with isobaric tagging. J Proteome Res. 2011;10(11): 5242-50.

50. Platten $\mathrm{M}$, et al. Tryptophan metabolism as a common therapeutic target in cancer, neurodegeneration and beyond. Nat Rev Drug Discov. 2019;18(5): 379-401.

51. Murakami S. Role of taurine in the pathogenesis of obesity. Mol Nutr Food Res. 2015;59(7):1353-63.

52. Mortada I. Hyperuricemia, type 2 diabetes mellitus, and hypertension: an emerging association. Curr Hypertens Rep. 2017;19(9):69.

\section{Publisher's Note}

Springer Nature remains neutral with regard to jurisdictional claims in published maps and institutional affiliations.

Ready to submit your research? Choose BMC and benefit from:

- fast, convenient online submission

- thorough peer review by experienced researchers in your field

- rapid publication on acceptance

- support for research data, including large and complex data types

- gold Open Access which fosters wider collaboration and increased citations

- maximum visibility for your research: over $100 \mathrm{M}$ website views per year

At BMC, research is always in progress.

Learn more biomedcentral.com/submissions 